\title{
Zustandsüberwachung von Motoren mit „smarten Sensor-Tags“
}

\author{
S. Wildermuth ${ }^{1}$, U. Ahrend ${ }^{1}$, C. Byner ${ }^{1}$, M. Orman ${ }^{2}$ \\ ${ }^{1} A B B$ AG, Forschungszentrum Deutschland, Ladenburg \\ ${ }^{2} A B B$ Ltd, Corporate Research China, Shanghai
}

\begin{abstract}
Zusammenfassung
Mikrosensoren bieten für die Zustandsüberwachung industrieller Anlagen drei attraktive Vorteile: kleine Baugröße, niedrige Kosten und geringe Leistungsaufnahme. Daher könnten sie eine flächendeckende Überwachung von industriellen Anlagen ermöglichen und so einen wichtigen Baustein für das Internet der Dinge darstellen. Weiterhin würde das Management ganzer Anlagenflotten zu akzeptablen Kosten ermöglicht. Allerdings wurde bisher eine großflächige Anwendung von beispielsweise MEMS Sensoren durch die sehr anspruchsvollen Umgebungsbedingungen in Industrieanlagen verhindert. In unserer Arbeit nutzen wir einen dreiachsigen geomagnetischen MEMS-Sensor, um Niederspannungsmotoren zu überwachen. Die Messperformance des Sensors wurde unter Bedingungen studiert, die denen in einer industriellen Anlage entsprechen. Weiterhin wurde das Magnetfeld eines ordnungsgemäß funktionierenden Motors mit dem Feld eines Motors mit gebrochenem Käfigläufer verglichen. Durch Frequenzanalyse der Messdaten war eine Unterscheidung der beiden Motorzustände möglich.
\end{abstract}

Keywords: Magnetfeld-Sensor, Zustandsüberwachung, elektrischer Motor, Frequenzanalyse, Internet der Dinge

\section{Einführung}

Die Zustandsüberwachung von industriellen Anlagen ist für Betreiber wichtig, um Stillstandzeiten und eine damit verbundene Reduzierung der Anlageneffektivität zu vermeiden. Viele verschiedene Methoden zur Zustandsüberwachung, insbesondere von elektrischen Motoren, wurden in den letzten Jahrzehnten untersucht, beispielsweise basierend auf Vibrationsanalyse oder auf Messungen von Motortemperatur und Motorströmen [1]. Einige werden heutzutage bereits routinemäßig an Motoren eingesetzt, die für den Anlagenbetrieb besonders wichtig sind. Ein weiterer vielversprechender Ansatz ist die Messung des Streumagnetfeldes eines Motors [2], weil diese Messung nicht invasiv und kontaktlos ist und somit einfach angewendet werden kann. Diese Messmethodik profitiert von der rasanten Entwicklung integrierter Magnetometer, z.B. für Anwendungen in Smartphones.

Die Aussagekraft der Messdaten eines Motors kann weiter erhöht werden, in dem eine ganze Flotte von Motoren mit "smarten Sensor-Tags“ ausgestattet wird $(A b b .1)$ und die damit gewonnenen Informationen mithilfe von statistischen Methoden analysiert werden [3]. Diese Tags enthalten Mikrosensoren, um Aussagen über Vibrationen, Temperaturen und Überlast- zustände des Motors zu ermöglichen. Moderne Verfahren der drahtlosen Kommunikation machen es möglich, dass die gesammelten Daten zusammengeführt und ausgewertet werden können, so dass ein Flottenmanagement mit Benchmarking und Überwachung der MotorEffizienz fabrikweit oder sogar unternehmensweit möglich wird. Cloud-basierte Systeme können zusätzliche Analyseschritte ermöglichen und die Ergebnisse auf einfachem Wege für Betreiber und Servicetechniker zugänglich machen. Die Grundlage für all diese zusätzlichen Anwendungen ist aber die lokal am Motor vorhandene Sensorik und Kommunikationsinfrastruktur.

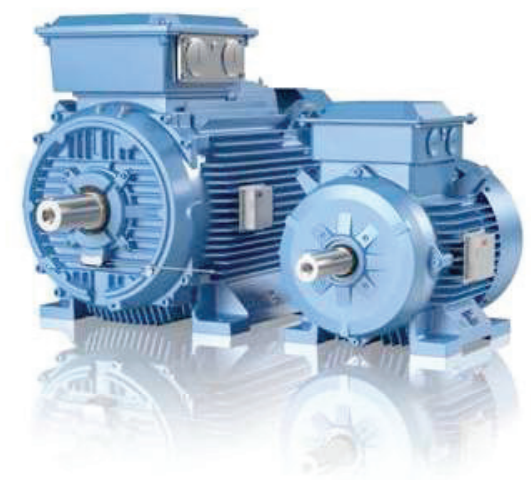

Abb. 1: „smarter Sensor-Tag“ an Niederspannungsmotoren 


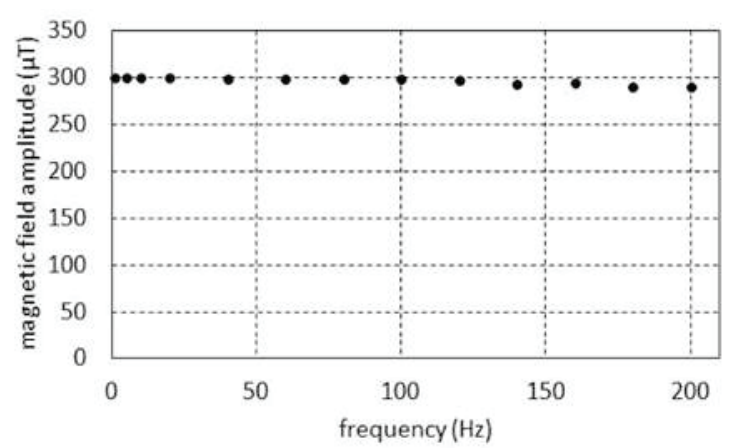

Abb. 2 Mit dem Magnetometer gemessene Amplitude in Abhängigkeit der Frequenz des zeitlich veränderlichen Magnetfeldes. Die Amplitude fällt zu hohen Frequenzen um 3,5\% $a b$.

Um einen weitverbreiteten Einsatz solcher Systeme zur Zustandsüberwachung zu ermöglichen, sind zwei Parameter von großer Wichtigkeit: Niedrige Kosten des Sensors kombiniert mit kleiner Baugröße. MEMSMagnetometer, wie sie heute beispielsweise in Fahrzeugen oder Smartphones zur Anwendung kommen, sind sehr klein (Baugrößen im Bereich einiger Millimeter), kostengünstig (weniger als 1 USD pro Sensor) und messen das Magnetfeld gleichzeitig in allen drei Raumrichtungen. Traditionell wurden zur Detektion von Magnetfeldern an Motoren sogenannte Flussspulen verwendet. Diese sind verhältnismäßig unhandlich und teuer, zudem ermöglichen sie die Messung des Magnetfeldes nur in einer Raumrichtung.

\section{Charakterisierung eines MEMS- Magnetometers}

Ein für den heutigen Konsumgütermarkt typisches MEMS-Magnetometer ist der BMC050 eCompass von Bosch Sensortec. Dieser integrierte drei-achsige Magnetfeldsensor bietet zur Messdatenerfassung eine direkte digitale Schnittstelle. Für erste Vorversuche wurde der Sensor mit einem nicht echtzeitfähigen NISystem ausgelesen, um den Frequenzgang des Sensors sowie die Messgenauigkeit und Reproduzierbarkeit der Messung zu ermitteln.

Der BMC050 kann mittels interner Triggerung mit einer Datenrate von $30 \mathrm{~Hz}$ betrieben werden. Externe Triggerung erlaubt die Datenrate auf bis zu $615 \mathrm{~Hz}$ zu erhöhen [4]. Für die geplante Zustandsüberwachung von elektrischen Motoren ist die Messung von zeitlich veränderlichen Magnetfeldern im Bereich von einigen $\mathrm{Hz}$ bis zu etwa $120 \mathrm{~Hz}$ besonders wichtig [5]. Daher wurde das Frequenzverhalten des Sensors bei einer Datenrate von $500 \mathrm{~Hz}$ vermessen $(A b b$. 2). Hierzu wurde ein WechselMagnetfeld mit einer konstanten Spitzenamplitude von $300 \mu \mathrm{T}$ im Bereich von $1 \mathrm{~Hz}$ bis zu $200 \mathrm{~Hz}$ variiert.

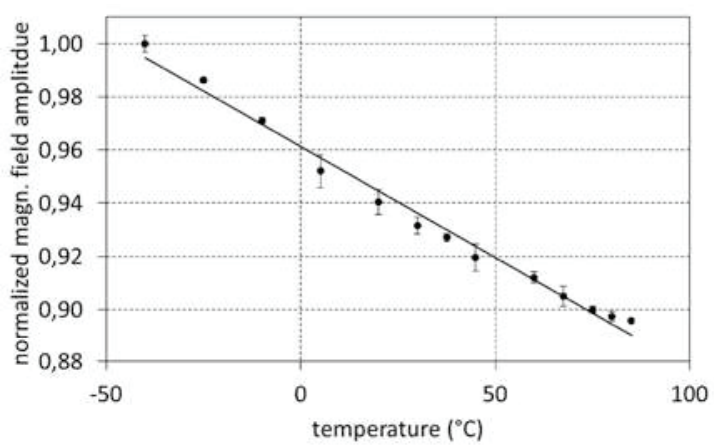

Abb. 3 Messsignal des Magnetometers in Abhängigkeit von der Umgebungstemperatur. Für den typischen Betriebsbereich in industriellen Anwendungen kann das Temperaturverhalten sehr gut durch einen linearen Zusammenhang angenähert werden.

Das Messsignal wich weniger als 3,5\% vom erwarteten Wert ab. Diese Messdaten können bei einer späteren Messung von Motormagnetfeldern verwendet werden, um das Frequenzverhalten des Sensors zu kompensieren.

Weiterhin wurde die Temperaturabhängigkeit des Magnetometers vermessen. Hierzu wurde das Magnetometer zusammen mit dem magnetfeldgenerierenden Stromleiter in einer Klimakammer betrieben. Die Temperatur der Klimakammer wurde von $-40^{\circ} \mathrm{C}$ bis $\mathrm{zu} 85^{\circ} \mathrm{C}$ variiert. Vor jeder Messung wurde die Temperatur in der Klimakammer für mindestens 1 Stunde konstant gehalten, um einen thermischen Gleichgewichtszustand zu erreichen. Wie erwartet, zeigt das Magnetometer eine nahezu lineare Temperaturabhängigkeit von $-8,7 \cdot 10^{-4} /{ }^{\circ} \mathrm{C}$ ( $\left.A b b .3\right)$. Dieses Messergebnis kann zur Kalibrierung dienen, um das Temperaturverhalten des Magnetometers bei einem späteren Einsatz im Industrieumfeld zu kompensieren.

\section{Magnetflussmessung an einem Nieder- spannungsmotor}

Das magnetische Streufeld eines Motors kann sowohl in axialer als auch radialer Richtung verwendet werden, um Fehlerfälle im Motor zu detektieren [2,5]. Das radiale Feld (senkrecht zur Motorwelle) wird in der Regel von Statorkern und Motorgehäuse abgeschwächt. Es wird im Wesentlichen vom magnetischen Fluss im Luftspalt beeinflusst. Das axiale Feld (parallel zur Motorachse) wird von den Strömen im Käfigläufer und in den Endwindungen des Stators erzeugt. Um beide Felder zu vermessen, muss das Magnetometer an verschiedenen geeigneten Stellen des Motors, beispielsweise nahe der Welle oder des Motorgehäuses, platziert werden. Da das hier untersuchte Magnetometer direkt drei Magnetfeldachsen vermisst, ist die exakte Ausrichtung des Sensors nicht nötig. 


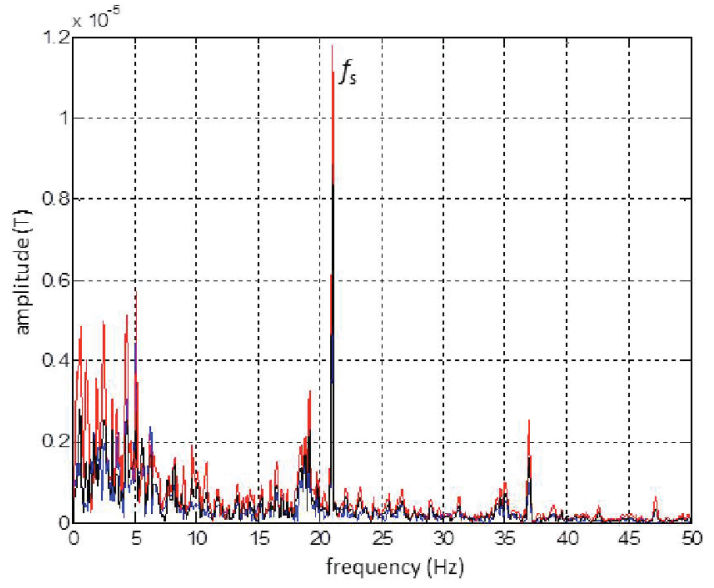

Abb. 4 Frequenzspektrum des Magnetfeldes des Motors in ordnungsgemäßem Zustand. Die Amplituden der Messung in den drei Raumrichtungen sind rot, schwarz, bzw. blau dargestellt.

Um Abweichungen von den normalen Betriebsparametern oder gar Fehlerfälle zu detektieren, ist es nötig den niederfrequenten Teil des Magnetfeldes zu vermessen [5]. Im Fall eines gebrochenen Käfigläufers spielt der Schlupfwert $s=\left(f_{s}-f_{r}\right) / f_{s}$ eine entscheidende Rolle, wobei $f_{s}$ die tatsächliche Drehfelddrehzahl (Netzfrequenz geteilt durch die Anzahl der Polpaare des Motors) und $f_{r}$ die tatsächliche Läuferdrehzahl ist. Weiterhin ist eine charakteristische Frequenz für einen gebrochen Käfigläufer die „broken bar“ Frequenz $\mathrm{f}_{\mathrm{bb}}=s f_{\mathrm{s}}$. [5]

Zwei Motoren der gleichen Bauart wurden mit dem Magnetometer vermessen: Ein Motor wurde in ordnungsgemäßem Zustand betrieben, wohingegen der andere Motor mit einem gebrochenen Käfigläufer präpariert wurde. Für diese Experimente wurden die Motoren mit einem Frequenzumrichter mit einer Frequenz von $21 \mathrm{~Hz}$ betrieben, so dass eine Rotationsgeschwindigkeit von 1241,4/min $(20,69 \mathrm{~Hz})$ erreicht wurde. Die Abtastrate des Magnetometers wurde auf $100 \mathrm{~Hz}$ eingestellt und Messdaten jeweils für $10 \mathrm{~s}$ aufgezeichnet.

Das Magnetfeld am Motor in ordnungsgemäßem Zustand wurde in allen drei Raumrichtungen vermessen $(A b b$. 4). Die Frequenzanalyse zeigt deutlich die Netzfrequenz von $21 \mathrm{~Hz}$ als dominierenden Beitrag in allen drei Magnetfeldrichtungen. Das gemessene Spektrum entspricht dem typischen Frequenzverhalten eines ordnungsgemäß funktionierenden Motors [5].

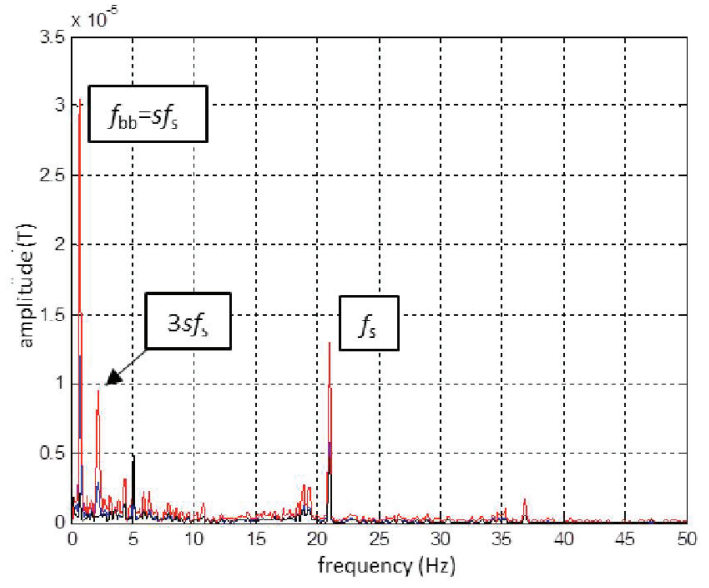

Abb. 5 Frequenzspektrum des Magnetfeldes des Motors mit gebrochenem Käfigläufer. Die Amplituden der Messung in den drei Raumrichtungen sind rot, schwarz, bzw. blau dargestellt.

Für den Motor mit gebrochenem Käfigläufer wurde das Magnetfeld ebenfalls vermessen $(A b b .5)$. Auch hier ist die Netzfrequenz wieder sichtbar. Allerdings ist deutlich erkennbar, dass in diesem Fall das Spektrum von niederfrequenten Anteilen dominiert wird. Aus den Messdaten wurde der Schlupfwert zu s=0,0345 und die Netzfrequenz zu 20,99 Hz ermittelt. Mit den oben angegebenen Formeln errechnet sich die charakteristische Frequenz $f_{b b} z u$ $0,724 \mathrm{~Hz}$ was mit den gemessenen Daten übereinstimmt. Eine weitere direkt im Spektrum identifizierbare Frequenz ist $3 s f_{s}$, die als zusätzlicher Indikator für einen gebrochenen Käfigläufer dienen kann [5]. Das Verhältnis der Amplitude der Frequenz $f_{b b}$ zu der RMSAmplitude des gesamten Spektrums könnte als Indikator für einen gebrochenen Käfigläufer verwendet werden.

\section{Zusammenfassung}

Magnetfeldmessungen mit einem MEMS Magnetometer wurden benutzt, um die charakteristische Signatur eines gebrochenen Käfigläufers im Frequenzspektrum des Magnetfeldes eines Niederspannungsmotors zu detektieren. Das Magnetometer wurde zuvor detailliert charakterisiert, um sicherzustellen, dass es für die Anwendung zur Motordiagnose geeignet ist. Insbesondere wurde das Frequenzverhalten des Magnetometers vermessen. Außerdem wurde die Signalstabilität bei variierenden Umgebungstemperaturen untersucht. Die Langzeitstabilität des Magnetometers wurde qualitativ untersucht und als ausreichend für die industrielle Anwendung eingestuft. 


\section{Ausblick}

MEMS-basierte Sensoren werden heute in vielen Massenprodukten, wie beispielsweise Smartphones, eingesetzt und sind daher sehr kostengünstig, hoch miniaturisiert und flexibel einsetzbar. Diese miniaturisierten Sensoren erlauben den Aufbau von kostengünstigen Messsystemen und ermöglichen eine Vielzahl von Anwendungen im industriellen Umfeld. Insbesondere die Zustandsüberwachung in industriellen Anlagen, wie beispielsweise von Motoren, aber auch weiteren Komponenten des Antriebsstrangs, ist von großem Interesse. In Kombination mit drahtloser Kommunikationstechnologie wie Bluetooth Low Energy wird eine schnelle und reibungslose Integration solcher Sensorsysteme auch im industriellen Umfeld möglich. Auf diese Weise wird die Überwachung einer ganzen Flotte von Anlagen und Maschinen technisch möglich und kommerziell interessant.

\section{Literaturnachweis}

[1] Tavner, P. J. "Review of Condition Monitoring of Rotating Electrical Machines", IET Electric power Applications, 2, pp. 215-247 (2008).

[2] Kokko, V. "Condition Monitoring of Squirrelcage Motors by Axial Magnetic Flux Measurement", PhD Thesis, University of Oulu (2003).

[3] Subbiah, S., Turrin, S., "Extraction and exploitation of R\&M knowledge from a fleet perspective", Proceedings IEEE Reliability and Maintainability Symposium (RAMS), pp. 1-6, (2015).

[4] Bosch Sensortec: Datenblatt, BMC050 Electronic Compass, (2011).

[5] Ceban, A., Pusca, R., Romary, R., "Study of Rotor Faults in Induction Motors Using External Magnetic Field Analysis", IEEE Transactions on Industrial Electronics, 59, pp. 2082-2093 (2012). 